the main therapy of SLE, has a long half-life. Thus, undetectable blood HCQ concentrations can be used to identify patients who do not take their treatment

Objectives: To identify the determinants of poor therapeutic adhesion in patients with SLE.

Methods: Case-control, retrospective, monocentric study. The cases were enrolled in our centre from $02 / 11 / 2011$ to $13 / 01 / 2015$ according to the following criteria: SLE defined according to ACR classification criteria and blood concentration of $\mathrm{HCQ}<100 \mathrm{ng} / \mathrm{ml}$ after a minimum of 2 months on therapy. For each case, the matched control was a lupus patient, enlisted from our centre the same week, with an HCQ dose greater than or equal to $800 \mathrm{ng} / \mathrm{ml}$. Case and control characteristics were compared using standard tests and a uni-multivariate logistic regression.

Results: One hundred and fifty patients were included, 75 cases ( 68 women) and 75 controls ( 72 women), with an average age of 35.7 years ( \pm 11.3 years) vs 35.6 years $( \pm 10.6$ years). Most patients had inactive lupus ( 3 patients had SLEDAI $\geq 4), 27 \%$ of them had benefited from therapeutic education sessions. The average dosage of $\mathrm{HCQ}$ was $1110 \mathrm{ng} / \mathrm{ml}$ within the control group. In our univariate analysis, nonadherent patients lived significantly further away from the centre than adherent patients (median distance [interquartile range]: $22^{11-52}$ vs $14 \mathrm{~km}$ [5.9-35], respectively, $\mathrm{p}=0.03$ ) and were more likely to be unemployed, (23 vs $8 \%$, respectively, $p=0.006$ ). Nonadherent patients had less often benefited from the patient's therapeutic education program (18 vs $35 \%$, respectively, $p=0.018$ ), were taking less treatment ( 3 vs 4 , respectively, $p=0.008$ ), had a significantly lower level of education ( $61 \%$ compared to $89 \%$ of patients with at least a bachelor's degree, $p<0.001)$. In our multivariate analysis, a level of education below the A levels was the strongest factor explaining poor therapeutic adherence, OR (IC 95): 4.09 (1.5-10.8).

Conclusions: The main drivers of therapeutic adherence during SLE are socioeconomic factors. The least educated and most disadvantaged patients are most likely to display poor therapeutic adherence. Targeted preventive actions and enhanced therapeutic education should be provided to them.

Disclosure of Interest: None declared

DOI: 10.1136/annrheumdis-2018-eular.5333

\section{FRI0338 HYDROXYCHLOROQUINE MAY HELP TO IMPROVE THE IN VITRO FERTILIZATION-EMBRYO TRANSFER OUTCOMES IN ANA AND DS-DNA POSITIVE PATIENTS}

F. Lian, J. Zhou, Y. Wang, H. Xu, X. Yang. Dept. Of Rheumatology, 1 St Affiliated Hospital Of Sun Yat-Sen University, Guangzhou, China

Background: Assisted reproductive technology has helped a large quantity of couples having trouble in natural pregnancy. Failure of in vitro fertilization-embryo transfer (IVF-ET) may be attributed to ANA and ds-DNA. ANA+/anti-dsDNA was related to low-quality embryos, low clinical pregnancy, and early miscarriage rate. $^{1}$ Hydroxychloroquine (HCQ) is recommended preconceptionally and throughout pregnancy for patients with SLE, and was proved to benefit the patients with Antiphospholipid syndrome. For those women with positive ANA and ds-DNA, but haven't any symptoms related to lupus or any other autoimmune diseases, the treatment for improving reproductive outcomes was controversial. In the present study, we retrospectively reviewed 156 patients with positive ANA and ds-DNA who underwent IVF-ET, compared the efficacy among different therapeutic strategies and observed side effects of the medication.

Objectives: To assess the efficacy, safety and tolerability of HCQ as preconceptionally and throughout pregnancy therapy in the treatment of IVF-ET patients with positive ANA and ds-DNA.

Methods: We retrospectively reviewed 156 patients in the treatment of IVF-ET with positive ANA and ds-DNA but without any symptoms in south China from January 2010 to December 2016 who received prednisone or prednisone $+\mathrm{HCQ}$ as preconceptionally and throughout pregnancy therapy. Prednisone was administrated at a dose of $7.5 \mathrm{mg} /$ day. HCQ was administrated at a dose of 0.2 twice a day. Details of the IVF-ET outcomes and side effects were collected.

Results: Of the 156 patients, no significance of demographic variables and reproductive related parameters such as duration of infertility, basal sex hormone, total Gn dose, E2 level on the day of HCG initiation, and number of retrieved oocytes was found among prednisone group (65 cases) and prednisone $+\mathrm{HCQ}$ group (91 cases). Fertilisation rate, implantation rate and clinical pregnancy rate were significantly higher in prednisone $+\mathrm{HCQ}$ group than in prednisone group, $75.8 \%$ vs $60.0 \%, p=0.017,29.7 \%$ vs $15.4 \%, p=0.032$, and $62.6 \%$ vs $47.7 \%, p=0.028$, respectively. Abortion rate was lower in prednisone $+\mathrm{HCQ}$ group, $7.0 \%$ vs $12.9 \%$, but not significantly. Clinical pregnancy rate was not associated the titers of ANA or ds-DNA. Low C3 was correlated with failure of implantation. None of the cases on prednisone or prednisone plus HCQ had side effects affecting the treatment course.
Conclusions: Combination of prednisone and HCQ may be more effective than solo treatment of prednisone for patients underwent IVF-ET with positive ANA and ds-DNA who had no clinical symptoms of autoimmune diseases.

\section{REFERENCE:}

[1] Fan J, Zhong Y, Chen C. Impacts of Anti-dsDNA Antibody on In Vitro Fertilization-Embryo Transfer and Frozen-Thawed Embryo Transfer. J Immunol Res. 2017;2017:8596181.

Acknowledgements: Project supported by Guangdong Natural Science Foundation ${ }^{\text {No. } 2016}$ A030313217).

Disclosure of Interest: None declared

DOI: 10.1136/annrheumdis-2018-eular.1626

\section{FRI0339 SLEDAI-2K RESPONDER INDEX-50 IS EFFECTIVE IN DEMONSTRATING PARTIAL RESPONSE IN A PHASE 2 , RANDOMISED PLACEBO-CONTROLLED STUDY OF USTEKINUMAB IN PATIENTS WITH ACTIVE SYSTEMIC LUPUS ERYTHEMATOSUS}

Z. Touma ${ }^{1}$, M.B. Urowitz ${ }^{1}$, D.D. Gladman ${ }^{1}$, C. Wagner ${ }^{2}$, B. Hsu², M. Chevrier ${ }^{2}$, S. Rose ${ }^{2}$, B. Zhou ${ }^{2}$, R. Gordon ${ }^{2}{ }^{1}$ Krembil Research Institute, Toronto, Canada; ${ }^{2}$ Janssen Research and Development, LLC, Spring House, USA

Background: Ustekinumab (UST), a monoclonal antibody that targets shared p40 subunit of cytokines IL-12 and IL-23, is being investigated in pts w/active sys temic lupus erythematosus(SLE). While traditional SLE Disease Activity Index 2000(SLEDAI-2K)scoring assesses complete SLE response for individual dis ease manifestations, SLEDAI-2K Responder Index-50(S2K RI-50)can be used to evaluate SLE responses using partial improvement $(\geq 50 \%)$ in each domain.

Objectives: To evaluate SLEDAI-2K vs S2K RI-50 response in a randomised, PBO-controlled trial of UST in pts w/active SLE.

Methods: We conducted a Ph2, PBO-controlled study in adults w/active disease (SLEDAI score $>6 \mathrm{w} />1$ BILAG A and /or $>2$ BILAG B scores)despite standardof-care therapy. $\mathrm{Pts}(\mathrm{n}=102)$ were randomised(3:2) to UST IV $6 \mathrm{mg} / \mathrm{kg}$ or $\mathrm{PBO}$ at wk0, followed by SC inj of UST $90 \mathrm{mg}$ q8w or PBO beginning at wk8, both added to standard-of-care. We calculated S2K RI-50 response at wk24 using various thresholds to define response including decrease of at least 1,2,3,4,5, or 6 points from baseline(BL) in S2K RI-50 score. We also compared proportion of pts w/SLEDAI-2K response vs S2K RI-50 response in pts receiving UST( $n=62)$ vs PBO $(n=40)$ at wk24.

Results: Change from BL SLEDAI-2K and S2K RI-50 scores were strongly correlated $(R=0.89, p<0.0001)$ at wk24. A greater proportion of UST vs PBO pts achieved S2K RI-50 response at wk24, regardless of threshold used to define response (table 1). The greatest differences in S2K RI-50 response rates between UST vs PBO were observed for a 4-point decrease $(23.1 \%, p=0.010)$, a 5 point decrease $(26.8 \%, p=0.010)$, and 6 -point decrease $(25.5 \% ; p=0.016)$ from $B L$. S2K RI-50 captured more responders than SLEDAI-2K at wk24, however, the difference in SLEDAI-2K 4-point response in UST vs PBO was $\triangle 27 \%(p=0.005)$ while $S 2 K$ Rl-50 was $\triangle 23 \%(p=0.010)$.

Abstract FRI0339 - Table 1. S2K RI-50 response rates at Wk 24 for various thresholds to define response

\begin{tabular}{lcccc}
\hline $\begin{array}{l}\text { Decrease from } \\
\text { Baseline }\end{array}$ & UST $(\%)^{\mathrm{a}, \mathrm{b}}$ & $\mathrm{PBO}(\%)^{\mathrm{a}, \mathrm{b}}$ & $\begin{array}{c}\text { Difference between UST and } \\
\text { PBO }\end{array}$ & p-value $^{\mathrm{c}}$ \\
\hline 1 Point Decrease & 96.0 & 94.2 & 1.8 & 0.3664 \\
2 Point Decrease & 90.0 & 84.2 & 5.8 & 0.2261 \\
3 Point Decrease & 86.9 & 74.1 & 12.8 & 0.0772 \\
4 Point Decrease & 86.2 & 63.1 & 23.1 & 0.0101 \\
5 Point Decrease & 74.5 & 47.7 & 26.8 & 0.0102 \\
6 Point Decrease & 66.3 & 40.8 & 25.5 & 0.0158 \\
\hline
\end{tabular}

Note: S2K Rl-50 response is defined differently in each row using different cutoffs.

S2K RI-50 uses partial response definition of $\geq 50 \%$ improvement for each individual SLEDAI-2K descriptor

${ }^{a}$ Values for pts meeting treatment failure criteria are set to missing from point of treatment failure forward.

${ }^{\mathrm{b}}$ Response based upon multiple imputations for missing data from Wk16 to Wk24, where Markov chain Monte Carlo method is used to make missing pattern monotone and serial logistic regression is used to impute monotone missing. The imputation model includes treatment group and baseline SLEDAI-2K covariate.

Test for greater treatment effect in UST over PBO (alternative hypothesis) is based upon logistic regression w/treatment group, baseline SLEDAI-2K, baseline medication use for SLE and race as covariates.

Conclusions: S2K RI-50 is an instrument that can capture partial clinically important improvement of $\geq 50 \%$ in SLE disease manifestations. The data suggests 
cutpoints for defining S2K RI-50 response in clinical trials of pts w/moderatesevere SLE disease activity.

Disclosure of Interest: Z. Touma Grant/research support from: Janssen Research and Development, LLC, M. Urowitz Grant/research support from: Janssen Research and Development, LLC, D. Gladman Grant/research support from: Janssen Research and Development, LLC, C. Wagner Employee of: Janssen Research and Development, LLC, B. Hsu Employee of: Janssen Research and Development, LLC, M. Chevrier Employee of: Janssen Research and Development, LLC, S. Rose Employee of: Janssen Research and Development, LLC, B. Zhou Employee of: Janssen Research and Development, LLC, R. Gordon Employee of: Janssen Research and Development, LLC DOI: 10.1136/annrheumdis-2018-eular.4877

\section{FRI0340 DOSAGE OF HYDROXYCHLOROQUINE (PLAQUENIL) ONLINE SURVEY BY PATIENT ORGANISATION NVLE}

Z. Osmani, W. Zacouris-Verweij, S. Otteron behalf of Lupus APS committee. Lupus APS committee, NVLE patient association, Utrecht, Netherlands

Background: Hydroxychloroquine (HCQ) has been proven effective in several immune mediated diseases. Long-term use of $\mathrm{HCQ}$ is very common in patients with systemic autoimmune disease. The greatest advantage of HCQ is that it may reduce the risk of flares and thereby allow glucocorticoid dose reduction. The risk/ benefit ratio of $\mathrm{HCQ}$ is excellent but $\mathrm{HCQ}$ is also known for its potentially severe and fortunately rare side effect: retinal toxicity. In The Netherlands, there are no standard guidelines regarding the dosing of $\mathrm{HCQ}$ and ophthalmologic screening of HCQ-induced complications. The American Academy of Ophthalmology (AAO) has recently published their revised recommendations on screening and dosing of $\mathrm{HCQ}$. Risk of retinal toxicity is mainly determined by the two most significant risk factors: daily dose of $\mathrm{HCQ}(\mathrm{mg} / \mathrm{kg} /$ day) and duration of $\mathrm{HCQ}$ therapy (years). The AAO recommends a maximum daily $\mathrm{HCQ}$ dosage of $<5.0 \mathrm{mg} / \mathrm{kg}$ real weight, to reduce the risk of toxicity. ${ }^{\text {Marmor, } 2016}$

Objectives: To raise more attention for the revised recommendation of the AAO, our patient organisation started an online survey asking patients with (systemic) autoimmune diseases which dose of $\mathrm{HCQ}(\mathrm{mg})$ they take on a daily basis.

Methods: Patients in the Netherlands were given the opportunity to complete the online survey at the website of the NVLE (Dutch patient association for people with Lupus Erythematosus, Anti-Phospholipid Syndrome, Systemic Sclerosis and Mixed Connective Tissue Disease) from July 26th - November 18th, 2017. The promotion of the survey took place solely through Social Media with a link to the survey. A total of 24 questions had to be filled in to complete the survey. Each individual was asked to write down their real weight $(\mathrm{kg})$ for calculating the daily $\mathrm{HCQ}$ dosage (mg/kg/day).

Results: A total of 705 individuals completed the online survey. The daily dosage (mg/kg/day) was available from 645 patients. The majority were females $(n=645)$ and diagnosed with (systemic) lupus erythematosus $(n=518)$. The average dosage of $\mathrm{HCQ}$ was $4,50 \pm 1,68 \mathrm{mg} / \mathrm{kg} /$ day. The daily dosage of $5 \mathrm{mg} / \mathrm{kg}$ was exceeded by 258 of the patients (40\%). Eighty-one individuals $(12.6 \%)$ used a daily dosage of $>5 \mathrm{mg} / \mathrm{kg}$ for more than 10 years. The most reported HCQinduced complications were gastrointestinal complaints $(n=55)$, problems with vision $(n=43)$, and nausea $(n=32)$.

Conclusions: Patients (and prescribing physicians) should be informed about risk of toxicity, proper dose levels, and the importance of regular annual screening. Physicians prescribing HCQ must aim for a daily HCQ dosage of less than $5.0 \mathrm{mg} / \mathrm{kg} / \mathrm{day}$, especially for patients using HCQ for already more than 10 years. (Inter)national guidelines regarding the screening and dosing of HCQ should be provided by the authorities to secure patient safety and reduce the frequency of (severe) retinal complications.

\section{REFERENCE:}

[1] Michael F, Marmor, et al. Recommendations on Screening for Chloroquine and Hydroxychloroquine Retinopathy (2016 Revision). Ophthalmology 2016;123(6):1386-94.

Acknowledgements: We would like to thank prof. dr. Frank van den Hoogen for his advice and dr. Els van de Ende for reading our abstract.

Disclosure of Interest: None declared

DOI: 10.1136/annrheumdis-2018-eular.2495

\section{FRIDAY, 15 JUNE 2018}

SLE, Sjögren's and APS - clinical aspects (other than treatment)

\begin{tabular}{l|l}
\hline FRI0341 & LUPUS LOW DISEASE ACTIVITY STATE (LLDAS) \\
& VERSUS CLINICAL REMISSION AS TREATMENT \\
& TARGETS IN THE FIRST 18 MONTHS OF SYSTEMIC \\
& LUPUS ERYTHEMATOSUS MANAGEMENT
\end{tabular}

A. Floris, M. Piga, G. Cappellazzo, E. Chessa, M. Congia, A. Cauli, A. Mathieu. Chair of Rheumatology and Rheumatology Unit, University and AOU of Cagliari, Monserrato, Italy

Background: In the view of applying a treat to target (T2T) strategy in the management of systemic lupus erythematosus (SLE), a novel definition of minimal acceptable disease activity - the lupus low disease activity state (LLDAS) ${ }^{[1]}$ - and clinical remission $(\mathrm{CR})^{[2]}$ were recently proposed.

Objectives: To compare attainability and outcomes of LLDAS and CR as treatment targets in the early stages of SLE management.

Methods: LLDAS and CR prevalence were analysed at 6 (T1) and 18 (T2) months after treatment initiation (TO) in a monocentric cohort of 107 (median disease duration 9.7 months) prospectively followed Caucasian SLE patients. LLDAS was defined as SLE disease activity index 2000 (SLEDAI-2K) $\leq 4$ without major organ activity and no new disease activity, physician global assessment (03) $\leq 1$, prednisone $\leq 7.5 \mathrm{mg} /$ day and well-tolerated immunosuppressant dosages. CR was defined as clinical SLEDAI-2K $=0$ (increased anti-dsDNA and low complement were excluded) and prednisone $\leq 5 \mathrm{mg} /$ day in patients treated with/without stable immunosuppressants and/or antimalarials. Multivariate models were built to identify factors associated with failure to achieve LLDAS and CR, as well as to investigate relationship between the latter and early damage accrual (defined as SLICC/Damage Index $\geq 1$ at T2).

Results: LLDAS was achieved significantly more frequently than CR both at $\mathrm{T} 1$ [47 (43.9\%) vs. 25 (23.4\%); p<0.001] and T2 [48 (44.9\%) vs. $35(32.7 \%)$ $\mathrm{p}<0.001]$. Out patients achieving LLDAS, $25(53.2 \%)$ and $35(66.7 \%)$ concomitantly fulfilled the criteria for $\mathrm{CR}$ at $\mathrm{T} 1$ and $\mathrm{T} 2$, respectively. A prednisolone (PDN) dose exceeding the minimal acceptable range set in the respective definitions was the most frequent reason for failure to achieve both LLDAS and CR (in $83.0 \%$ of no-LLDAS patients at T1\% and $95.1 \%$ of no-CR at T1). The disease manifestations with the highest persistence rate during follow-up were: increased antidsDNA (persistently present in $85.7 \%$ and $67.5 \%$ of cases at T1 and T2, respectively), low complement $(73.2 \%$ and $66.3 \%)$ and renal abnormalities $(46.4 \%$ and $28.6 \%$ ). Renal involvement at baseline significantly associated with failure to achieve LLDAS both at T1 (OR: 7.8, 95\% Cl: 1.41-43.40; $\mathrm{p}=0.019)$ and T2 (OR: 3.87, 95\% Cl 1.41-10.6; $\mathrm{p}=0.008$ ) and $\mathrm{CR}$ at T2 (OR 9.46, 95\% Cl 1.13-78.8). High PDN dosage was significantly associated with no-CR achievement both at T1 (OR 6.23, 95\% Cl 2.2-18.3; $\mathrm{p}=001$ ) and T2 (OR 1.11, 95\% Cl 1.02-1.20; $\mathrm{p}=0.02)$. Early damage was recorded in $23(21.5 \%)$ patients and was significantly associated, on multivariate analysis, with older age at diagnosis (OR $1.0595 \% \mathrm{Cl}$ 1.01-1.09; $p=0.016$ ) and failure to achieve LLDAS (OR 4.82, 95\% Cl 1.44-16.09; $p=0.11$ ) at $T 1$. No significant association was found between early damage and failure to achieve CR, possibly due to its low prevalence among this cohort of early SLE patients.

Conclusions: During the early stages of SLE, LLDAS and CR overlap definitely less frequently than reported in long-standing disease $(53.2 \%-66.7 \%$ vs $83.9 \%$ $\left.96.5 \%{ }^{[2]}\right)$. Although remission is recommended as the primary treatment target in SLE, LLDAS may represent a valid alternative in the first stages of disease management, being more attainable compared to $\mathrm{CR}$ and negatively associated to early damage.

REFERENCES:

[1] Franklyn K, et al. Ann Rheum Dis 2016;75:1615-21.

[2] Zen M, et al. Ann Rheum Dis 2017;76:562-5.

Disclosure of Interest: None declared

DOI: 10.1136/annrheumdis-2018-eular.2774 